\title{
EMOTION-I Model: A Biologically-Based Theoretical Framework for Deriving Emotional Context of Sensation in Autonomous Control Systems
}

\author{
David Tam ${ }^{*}$
}

Department of Biological Sciences, University of North Texas, Denton, Texas 76203, USA

\begin{abstract}
A theoretical model for deriving the origin of emotional functions from first principles is introduced. The model, called "Emotional Model Of the Theoretical Interpretations Of Neuroprocessing", abbreviated as the "EMO$T I O N$ ", derives how emotional context can be evolved from innate responses. It is based on a biological framework for autonomous systems with minimal assumptions on the system or what emotion is. The first phase of the model (EMO$T I O N-I)$ addresses the progressive abstraction of the sensory input signals within relevant context of the environment to produce the appropriate output actions for survival. It uses a probabilistic feedforward and feedback neural network with multiple adaptable gains, self-adaptive learning rate and modifiable connection weights to produce a self-organizing, selfadaptive system incorporating associative reinforcement learning rules for conditioning and fixation of circuitry into hardwire to form innate responses such that contextual feel of sensation is evolved as an emergent property known as emotional feel.
\end{abstract}

Keywords: Origin of emotions, autonomous control system, model of emotion, neural processing, contextual representation, sensation.

\section{INTRODUCTION}

Emotion is one of the most studied subjects in many disciplines of science including psychology, physiology, philosophy, anthropology, etc., and recently in robotics. Yet, it is one of the most controversial subjects because of the differences in definition, in perception, and in perspectives, among other things such as introspection and retrospection by human cognition. Rather than engaging in the debate of whether emotion is unique to human, whether other animals or robots could have emotions, this paper focuses on deriving the emergent property called emotion from the basic principles required for processing autonomous control functions. We will use an inter-disciplinary approach that includes mathematics, neuroscience, physiology, psychology and engineering control science in the derivation of emotion. Due to the volume of research on the topic, we will limit our discussion on relevant classical literature in the derivation of emotions.

\section{EVOLUTIONARY APPROACH}

This approach to study the emergence of emotion in a self-actuating autonomous system is analogous to the approach to study how emotions are evolved in the biological system at the theoretical level rather than studying it at the phenomenological level. It is an evolutionary approach to derive the necessity for the emergence of an entity called "emotion" for processing sensory signals and its internal functions in order to survive in the real world. It is based on the computational principles needed for an autonomous robot to function in the real world without any external guide or control.

*Address correspondence to this author at the Department of Biological Sciences, University of North Texas, Denton, Texas 76203, USA;

E-mail: dtam@unt.edu
We consider a self-actuating autonomous robot as an animal (or organism) without any pre-programmed ability (i.e., a priori knowledge) to interact with the physical world. The task of this autonomous robot (or animal) is to derive its own working principles to interact with the real world with a minimal set of assumptions, and see how emotions are evolved in this process as the necessary condition to survive in the real world, without assuming what emotions are or should be.

\section{OPERATIONAL APPROACH}

By using this approach of inquiry, we will bypass the unavoidable debate on the human perception of what emotions are, what they are used for, whether they are unique to humans, whether they exist in animals or robots, and any other subjective perception of what emotions are, including the debate of the subjective definitions of different emotions. In other words, we will use an objective approach to study this phenomenon (called emotion) without assuming its functional role in animals, humans or robots. Rather, we will study the phenomenon of autonomous control in animals, and observe what principles of operation are required to survive in the real world. From these operational principles needed for survival, we will identify which of these governing principles happen to correspond to the entity that people identify as "emotion".

\section{ROBOTIC EMOTIONS}

Emotions in autonomous robots have been implemented recently in various systems, primarily as a study of autonomous behavior augmented by emotional controls [1-3]. Most often, the autonomous robots mimic the human emotions as an "add-on" to the unemotional cognition by introducing emotion as part of the process-control function rather than deriving emotional functions or exploring the origins of emotions in self-adaptive behavior. Although comparison be- 
tween the human cortical system and robots were made to characterize whether robots have emotions [4], such comparison does not address the functional role played by emotions in self-adaptive autonomous control or independent agent such as an animal.

\section{CYBERNETIC APPROACH}

We will derive a theoretical model of emotional functions from the first principles for autonomous control. A cybernetic system is an autonomous system that captures the essence of most basic biological and higher cognitive functions (including intellectual, emotional and mental functions). The derivation of this emotional model is based on capturing the minimal set of conditions that are fundamental to the survival (and/or appropriate interactions with the environment) of such autonomous systems.

\section{REAL WORLD INTERACTIONS}

It will be shown that within the framework of autonomous control, emotions emerge as natural phenomena in order for autonomous systems (animals or autonomous robots) to function appropriately within context in a real world environment. This foundation is based on the thesis that autonomous systems are independent agents (organisms or robots) that rely on an internal representation model of the external world to function accordingly.

\section{ROLE OF CENTRAL NERVOUS SYSTEM}

The role of the nervous system (either central nervous system (CNS) in animals or neural control system in robots) is to provide an accurate abstract representation of the external environment internally. Most importantly, this internal representation is not necessarily an exact replica of the external world, but an accurate contextual representation such that the autonomous system can respond appropriately under any given circumstances for survival and other operating functions.

One of the many schemes for capturing this abstract, contextual representation of the external environment is creating an internal model of the external world by the nervous system.

\section{INTERNAL MODEL OF EXTERNAL WORLD}

One of the advantages of creating an internal model of the external world is that it not only provides a contextual representation of the outside world, but also provides the prediction of how its future actions may have on the environment. It is this predictive power of the internal model that provides for what is known as "cognition" or "higher intelligence".

We will show that emotions, within this framework, correspond to the feedforward and feedback variables of the internal model used for assessing the accuracy of the model and its actions. Thus, emotions, in this perspective, are not necessarily unique to humans or animals, nor are they introspective constructs labeled/constructed by human to explain some psychological phenomena.

\section{EMOTION-I Model}

This model is called the "Emotional Model Of the Theoretical Interpretations Of Neuroprocessing", abbreviated by the acronym as the "EMOTION" model. This paper focuses on the first phase of development of the biological framework for this model: EMOTION-I. It addresses the emergence of the "feel" of sensation for increasing the chance of survival as the first step in internal pre-processing of emotions. The subsequent paper [5] will focus on the second phase in developing the minimal set of basic emotions for this model: EMOTION-II. It addresses the emergence of a metric for assessing the accuracy of the internal model. This internal model congruency consistency-check is represented by "emotion".

\section{AUTONOMOUS CONTROL SYSTEMS}

An autonomous control system is a self-actuating system capable of performing sensorimotor functions based on its internal controls. Most often, it is capable of decision making without external guide or control. Examples of autonomous systems are animals and autonomous robots.

Biological organisms (animals, in particular) can be considered as autonomous control systems because they are capable of performing sensory and motor functions independent of an external agent. Autonomous robots can also be considered as autonomous systems since their sensorimotor functions are controlled by their internal processors without relying on any external control.

\section{SOCIAL SYSTEMS}

Self-actuating autonomous systems are self-contained entities that operate independently. Although social systems of these individuals may be dependent on each other for social interdependency (such as a school of fish, an army of ants or a swamp of robots), the analysis of social interdependency is beyond the scope of this paper.

\section{COMPONENTS OF AN AUTONOMOUS SYSTEM}

Autonomous systems are self-contained entities that are composed of systems of many interacting parts, including sensory units, motor units and processing/controlling units. Together, they form a system exhibited as an animal (in biological systems) or a robot (in robotic systems).

\section{SURVIVABILITY AND APPROPRIATENESS OF AC- TIONS}

The task of the system is to integrate the sensory inputs by the internal processing units to produce output actions that are appropriate in the environment it lives in. The appropriateness of these output actions is determined by the accuracy of the internal model that produces actions for the organism to survive in its environment.

\section{REFLEX AS A SIMPLE AUTONOMOUS SYSTEM}

One of the simplest autonomous systems is the reflex system, which is endowed with sensory input, motor output 
and its processing elements that associate sensory input with motor output. The sensorimotor function is the minimal set of functions to be considered as autonomous system.

\section{STIMULUS-RESPONSE FUNCTON}

Mathematically, the sensory stimulus is considered as the input, $x$, which often encodes the intensity of the stimulus. The motor response is considered as the output, $y$, which often encodes the magnitude of the response. This sensorimotor function is sometimes called "stimulus-response function" (S-R function) in physiology.

\section{INPUT/OUTPUT (I/O) FUNCTION}

This stimulus-response function also corresponds to the mathematical input/output (I/O) function, $f$ :

$y=f(x)$

The input, $x(t)$, and output, $y(t)$, are often functions of time, $t$, i.e., time-varying functions; thus, the $\mathrm{I} / \mathrm{O}$ function, $f$, becomes:

$y(t)=f(x(t))$

Although, in general, the input and output can take on any real number $(x, y \in \mathfrak{R})$, it is advantageous to simplify the subsequent derivation using the range of $x$ and $y$ that is positive (i.e., $x \geq 0$ and $y \geq 0$ ), since negative values can reverse the direction (sign) of the computed I/O function unintentionally.

Therefore, the task of an animal is to produce an appropriate $\mathrm{I} / \mathrm{O}$ function such that the resulting action (motor output) will be an appropriate response in a given the environment (encoded by the sensory inputs).

\section{REFLEX ACTION}

Reflex is one of the simplest (most basic) sets of sensorimotor functions found in animals. The reflex-action found in animals is that, given a stimulus of sensory input, the animal is able to respond with a motor output independently without any external control. The response in a reflex-action is usually stereotypical for a given stimulus. It usually provides a physiologically appropriate response for the given stimulus that enables the animal to respond rapidly without needing higher-level processing. This usually increases the survivability of an animal by decreasing its response time.

\section{I/O MAPPING}

Mathematically, reflex is essentially an I/O mapping function that maps the input into the output space. This mapping function (Eq. 1) is usually a simple function for reflexes. It can be a linear function or a nonlinear function depending on the specific reflex.

Most of the physiological reflexes are nonlinear functions (often a sigmoidal function), in which there is a linear portion in the middle called the "physiological range", below which the sensory signal is too small to be sensitive to respond to, and above which the response is maxed out due to physical limitations of the response system.
The response in the physiological range is often a linear function, since it approximates the linear response range. Most reflexes operate at this linear region, although the physiological range of some reflexes may be exponential or logarithmic instead of linear when signal compression is required for efficient scaling. Pupil constriction reflex is an example.

\section{PHYSIOLOGICAL RANGE}

For a simple linear reflex system, the $\mathrm{I} / \mathrm{O}$ function is given by:

$y(t)=a \cdot x(t)+b$

where $a$ and $b$ are constants.

For a nonlinear reflex system, such as a sigmoidal response, the I/O function can be represented by:

$$
y(t)=\frac{1}{1+e^{-a \cdot x(t)}}
$$

with an approximately linear response at the physiological range.

\section{LOOK-UP TABLE}

Because these I/O functions are rather simple, which map the input space into the output space by some straightforward mapping functions (or look-up tables), these basic reflexes are usually not considered as representing any "higherfunctions" such as emotion, cognition, perception or intelligence. Higher-level processing often requires a much more complex I/O function, and it is often dependent on additional parameters and conditions.

\section{HIGHER-LEVEL MAPPING}

We will derive the I/O function that leads to the emergence of emotions from the additional parameters and conditions that are beyond this basic set of simplistic reflex functions. Thus, emotions and higher-cognition are an expansion of this basic reflex I/O function that maps the input space into the output space depending on other additional factors. The higher-level processing/controlling functions, such as perception, cognition, emotion and intellectual functions, are the additional attributes of the system that allow them to perform above and beyond the basic sensorimotor reflex functions.

\section{PROBABILISTIC STOCHASTIC I/O FUNCTIONS}

The I/O function for a reflex can also be either deterministic or non-deterministic (i.e., probabilistic). If it is deterministic, the exact response can be determined by the $\mathrm{I} / \mathrm{O}$ function, such as Eqs. 3 and 4. If the reflex is nondeterministic, Eq. 3 can be re-represented by a probabilistic function:

$$
y(t)=\operatorname{Prob}(a \cdot x(t)+b)
$$

which takes on the normalized value of $[0,1]$.

\section{PHYSIOLOGICAL NOISE}

In the real world, any physical system is inherent with noise, which provides the basis for a probabilistic system. Eq. 
5 can be implemented equivalently by an addition of random noise function:

$$
y(t)=a \cdot x(t)+b+\operatorname{Noise}(\sigma)
$$

where $\operatorname{Noise}(\sigma)$ can be any real valued random number drawn from any random distribution with a variance of $\sigma$ according to the implementation details. Similarly, the nondeterministic form of Eq. 4 can be represented by:

$$
y(t)=\operatorname{Prob}\left(\frac{1}{1+e^{-a \cdot x(t)}}\right)
$$

or implemented by an addition of random noise function:

$$
y(t)=\frac{1}{1+e^{-a \cdot x(t)}}+\operatorname{Noise}(\sigma)
$$

\section{SOURCE OF NOISE}

The source of noise may come from the sensory signal, the transfer function or the output element. For simplicity, without loss of generality, since most noises are additive, we will collapse these noise sources into a single noise term in the output element, $y(t)$, in Eqs. 6 and 8. For non-additive noise, a separate noise term can be added to each of the sensory input function, transfer function, and motor output function.

\section{EXPLORATION IN LEARNING}

The advantage of using a probabilistic function instead of a deterministic function is that it allows for variations in output response for the same stimulus. This variability is important in both learning and evolution, which require a trial-anderror approach to explore the unknown parameter space.

The probabilistic response essentially provides the variations (or randomizations) needed for exploration in learning and in evolution. If the response is too deterministic, no variations will result, and the animal will always produce the same response as in a typical reflex, which is always the same for a given stimulus intensity.

\section{SELF-EXPLORATION}

Self-exploratory adaptation/learning and evolution may not occur without variability. Thus, probabilistic response function is essential in self-adaptive systems whereby the variability in output can be used as a feedback signal for evaluating the adaptability of the system in response to different exploratory actions. In other words, it enables the system to explore the parameter space autonomously, similar to applying the Monte Carlo simulation method but applying in the real world in this case.

\section{MODIFICATION OF REFLEX ACTIONS}

Advanced behaviors (behavioral responses) often require atypical responses instead of stereotypical responses. This often requires modification of the response found in learning and in evolution. Modification of the response implies changing the $\mathrm{I} / \mathrm{O}$ function.

\section{SENSITIZATION}

Reflex can be modified to adapt to the environment, such as increasing or decreasing the response amplitude. When the response is increased over time (over repeated trials), it is called "sensitization" in physiology. When a reflex is sensitized to a stimulus, it leads to a larger response amplitude. This is essentially an amplification of the response by increasing the scale of the I/O function.

That is, Eq. 1 can be modified by a scaling factor, $c$, (or gain) such that the output becomes:

$y=c \cdot f(x)$

where $c>1$ for sensitization.

\section{HABITUATION}

When the response is decreased over time (over repeated trials), it is called "habituation;" the reflex is habituated to the stimulus, i.e., it becomes less sensitive. When the reflex is habituated to the stimulus, it leads to a smaller response amplitude. Thus, habituation is sometimes called "desensitization" in physiology. In other words, the output becomes smaller:

$y=c \cdot f(x)$

where $c<1$ for habituation.

\section{GAIN CONTROL}

The increase or decrease in the scale factor, $c$, can also be considered as changing the "gain" of a control system such that the output is amplified or reduced for a given input. Thus, this physiological adaptation is essentially a gain control for the system to respond. In other words, the I/O function of the reflex can be altered rather than fixed. Thus, the system is an adaptive system in which the reflex can be altered by either sensitization or habituation.

\section{ADAPTATION - SINGLE-STIMULUS LEARNING}

Sensitization and habituation form the class of physiological learning called "adaptation". It is the simplest form of learning in which the response output of the reflex is either increased or decreased when the stimulus is repeated over many trials. Whether the response will increase (sensitize) or decrease (desensitize) is dependent on the context of the stimulus.

Note that adaptation is a type of learning that requires only a single stimulus. We will show that other types of learning will require two or three events to occur.

\section{CONDITIONS FOR ADAPTATION}

When the stimulus is potential harmful (noxious) to an animal, and when this stimulus is repeated over time, the adaptation often results in sensitization. When the stimulus is potential harmless (non-noxious) to an animal, and when this stimulus is repeated over time, the adaptation often results in desensitization or habituation.

Noxious stimulus is often derived from a painful sensory source, when pain serves as an alarm signal for an animal to respond to, and become sensitized to that signal. (Although we have not defined the emotional quantity called pain in our derivation so far, we include it in our discussion here to convey the contextual meaning of a sensation, i.e., how this sen- 
sory signal could be used in the I/O stimulus-response function. We will derive the emotion pain in the discussion later.)

\section{PHYSIOLOGICAL APPROPRIATENESS AND CON-} TEXTUAL MEANING

These changes in response amplitude are physiologically appropriate. Sensitization to the noxious stimulus allows the animal to respond more readily to prevent potential harm. In other words, this simple amplification of the reflex-response allows the animal to predict the future better by making the implicit assumption that amplifying the stimulus-response $\mathrm{I} / \mathrm{O}$ function could prevent future harm. Although this implicit assumption may not always apply in every unforeseen situation, it is a pragmatic solution in most physiological circumstances.

Conversely, when the repeated stimulus is potentially harmless (non-noxious), the animal would habituate to the stimulus. This is also physiologically appropriate because when the stimulus is non-noxious, the animal does not need to respond as intensely to the same stimulus to save energy.

\section{PHYSIOLOGICAL ADVANTAGES}

There are many physiological advantages of habituation to the non-noxious stimuli. It provides a minimization of the energy-cost in producing the reflex-action. When an animal is confronted with multiple stimuli that require coordination, competition and interference of different stimulus-responses could occur in a complex system. Reducing the response by habituation can reduce the chance of interference of reflexactions when the animal responds to multiple stimuli simultaneously.

\section{GILL-WITHDRAWAL REFLEX AS AN EXAMPLE}

The gill-withdrawal reflex studied in Aplysia is a classic example of the habituation (desensitization) of reflex in response to repeated non-noxious stimuli [6-8]. Intuitively, this adaptation corresponds to the interpretation that since the sea slug is constantly bombarded with stimuli from sea waves, those harmless stimuli can be ignored if they do not represent potential threats to the animal.

\section{FEEDFORWARD CONTROL}

In order to evaluate the "appropriateness" (or "survivability") of the above self-adaptive responses, feedback and/or feedforward control are often needed. The implicit assumption is that amplifying or reducing (sensitizing or habituating) the reflex-response to increase the survivability is a feedforward prediction. In other words, it does not rely on the feedback of the response to correct for its action. It merely produces an output action, which "projects" that the outcomes would be appropriate with an implicit assumption that it will, regardless of whether it will or not.

\section{FEEDBACK CONTROL}

In contrast, a feedback system takes its current output response (and other environmental factors) into account as part of the input to evaluate the next response action, whereas a feedforward system does not. So although a feedback control system may seem more appropriate in self-adaptation, a feedforward control system does have its usefulness in autonomous systems. It provides fast responses without needing the extra computational processing time overhead needed in a feedback system. Furthermore, a feedback control system can be susceptible to instability, such as oscillations, when the feedback signal is time-delayed. Thus, both feedback and feedforward systems do have their own advantages and disadvantages; they serve different purposes in the design and evolution of an autonomous self-actuating, selfadaptive system.

\section{CONTEXTUAL "FEEL" IN SENSATION}

The alteration of reflex-action by sensitization or habituation is based on the implicit assumption that the animal is able to project (predict) whether the stimulus is potentially noxious or not. Although the animal may not be considered as having any high-level conscious perception cognitively at this stage, the prediction of potential harm is crucial to the emergence of the contextual "feel" in sensation (i.e., the emotional content of a sensation - whether it feels "pleasant" or not).

\section{EMOTIONAL “QUALITY” IN SENSATION}

In other words, encoding merely the intensity of the stimulus in the input-function, $x(t)$, is not sufficient to recognize whether the stimulus is potentially harmful or harmless, which in turn translated into whether it is pleasant or not emotionally. The abstraction of the stimulus "quantity" into stimulus "quality" is the first step in the emergence of emotional "feel" in sensation (sensory input).

\section{PLEASANT/UNPLEASANT SENSATION}

Thus, the abstraction of the stimulus in terms of potential harm (harmfulness) requires the emergence of the contextual feeling in sensation. Although cognition may not exist at this low level of processing, nonetheless, the sensory stimulus is no longer interpreted in isolation by the system. Rather, it is interpreted based on the context in which the sensation is received relative to the projected/predicted survivability of the animal.

\section{EMOTIONAL CONTEXT AND SURVIVABILITY}

Signal is hypothetically interpreted as unpleasant if it is potentially noxious (or harmful) to the integrity/survivability of the animal. The most unpleasant form of sensation would become pain. Conversely, sensation is interpreted as pleasant if it is preserving the survivability of the animal.

This pleasantness of sensation becomes one of the most elemental forms of "contextual" sensation in the emotional context. The "context" is the survivability of the animal, the stimuli and environment in which it is interacting with.

\section{PHYSICAL SENSATION AND EMOTIONAL SENSA- TION}

In higher animals, such as mammals, emotional sensation is interpreted and processed at the thalamic level [9]. On the other hand, physical sensation that encodes the stimulus intensity (stimulus quantity) is encoded at the sensory receptorcell level. 


\section{PHYSICAL PAIN (HURT) AND EMOTIONAL PAIN (SUFFERING)}

Pain is a good example to describe the distinction between emotional sensation and physical sensation. There are two distinct components of pain as perceived in higheranimals - the emotional pain called "suffering" and the physical pain called "hurt" in sensation.

Hurt is the physical damage to the tissue, i.e., the stimulus intensity encoded by action potentials in the first-order neurons (pain fibers). Suffering is the emotional context in which the hurt is felt, i.e., how bad it feels - the quality of the sensation rather than the quantity of the sensation. The emotional component of sensation is processed by the thalamic nuclei.

\section{NEUROPHARMACOLOGICAL DISSOCIATION OF PHYSICAL PAIN FROM EMOTIONAL PAIN}

Physiologically, the emotional aspect and the physical aspect of the same nociception (pain sensation) can be separated by dissociative anesthetics, such as ketamine and PCP (phencyclidine), in which physical hurt can be felt without the emotional suffering in higher-animals. In other words, when an animal is under dissociative anesthetics, it can feel the physical pain (hurt sensation) but that hurt does not bother it emotionally, and is totally tolerable without feeling any suffering emotionally associated with that hurt.

Thus, physical pain and emotional pain are two distinct components of the same sensation for pain. The emotional component can be separated from the physical component physiologically by drugs, which demonstrates the existence of the emotional context of a sensation (sensory stimulus) in animals. This distinction between hurt and suffering (physical and emotional pain) can be separated physiologically and neurologically; thus suffering is not merely a psychological construct or subjective perception.

\section{FEEDFORWARD MODEL PREDICTION}

The emergence of contextual sensation is essential for the survival of the animal, which can predict the potential outcomes of the sensory stimulus with respect to the animal's survivability. This context takes into the account of environment and integrity (survivability) of the animal involved - a feedback control. It also presumes the ability to predict (or at least project) the outcomes so that it can produce the physiologically appropriate output - a feedforward control.

\section{CONTEXTUAL SENSATION}

An animal can be considered as forming a conceptual model of the world (or the environment in which it lives) and itself to produce this prediction (projection) accurately. This also implies forming an implicit "model" of the external world and a "model" of its own internal world where the sensorimotor processing is done.

\section{IMPLICIT MODEL}

Although this contextual model may not be formed explicitly, nonetheless, it can be considered as having an implicit conceptual framework to model a rudimentary model of the world around it. This model may not be a concrete model or an explicit model, but a conceptual model such that the behavior (motor output) is produced appropriately for a given sensory input in the physiological context, even though the animal may not even be considered as having any "concepts", "perception" or "model" of its own at this elementary stage of development because all these responses are merely reflexive responses without any higher-level processing or cognition.

\section{MECHANISTIC STEREOTYPICAL RESPONSES}

It is important to note that these reflexive actions are mechanistic responses (because they are very stereotypical) rather than cognitive emotional responses with higher-level processing or recognition at the awareness level. Yet, this implicit representation of contextual information provides the conceptual framework for the derivation of emotional response from first principles in relation to the survivability of an animal or any autonomous being.

\section{ABSTRACTING SIGNIFICANCE OF SENSORY SIG- NALS BY CONTEXT}

The above analysis of the emergence of "emotional feel" in sensation forms the basis for abstraction of sensory inputs by context. In other words, sensory inputs are no longer merely encodings of the stimuli intensity, which represent the physical sensation. Rather, sensory inputs are processed in such a way that it takes context into account to form an abstraction of the "significance" of the stimuli.

The significance of the sensory inputs is evaluated based on the context in which the inputs are received and interpreted. In the above example, the significance of the sensory inputs is evaluated based on the survivability of the animal. Thus, the emotional feel takes on the significance of that sensation for instructing the animal how to respond appropriately if it were to increase its likelihood of survival in its environment.

\section{OPERATIONAL DEFINITION OF EMOTIONAL SEN- SATION}

The above analysis provides the theoretical basis for the derivation of elementary emotions in sensation called "emotional feel" based on first principles rather than retrospection in psychology or fact-of-evolution in biology. Although these responses may be hardwired with some modifiability to some extent, they are still simple reflexes without any complex processing or cognition. They are merely simple reflexive responses that are governed by simple equations represented by the I/O functions similar to Eqs. 1-9.

Note that the emotional components in these I/O functions are implicit rather than explicitly represented. The implicit representation is the context in which it takes on in altering the response. This re-representation of the stimulusresponse can be considered as an elementary (first-level) emotional response as far as sensation is concerned. It provides the qualitative emotional feel even though the animal does not necessarily have any explicit emotions with respect to these reflexes. Based on this framework, we will explore the neural mechanisms for establishing this contextual sensation for an autonomous system. 


\section{CONDITIONED REFLEX}

It is well known that the stimulus of a reflex can also be "switched" to a different one other than the original stimulus. In such case, the reflex is called "conditioned reflex" because the response is altered by a conditioned stimulus.

For example, the eye-blink reflex is one of the classical experiments in which the air-puff stimulus that induces the eye-blink response can be switched over to a tone if a tone stimulus is paired with the air-puff stimulus [10]. A rabbit can learn (or be conditioned) to blink the eyes when the tone is presented instead of an air-puff to the eye.

\section{CLASSICAL CONDITIONING - TWO-STIMULI AS- SOCIATIVE LEARNING}

Classical (Pavlovian) conditioning (a well-known psychological phenomenon) is a mechanism in which two stimuli are paired to establish the association between the stimuli and response that were not established before. It requires two stimuli instead of one stimulus as in adaptation discussed earlier. The stimulus-response function of the original pair (the innate unconditioned stimulus (US) and unconditioned response (UR) pair) is transferred to the novel pair (conditioned stimulus (CS) and conditioned response (CR) pair).

Note that this type of learning requires two stimuli (US and CS) to form the association. The end-result is that the original I/O function between US and UR is changed such that the new I/O function is established between CS and CR.

\section{TRANSFER OF CONDITIONING STIMULUS}

This transfer is established by pairing the presentation of the unconditioned stimulus with the conditioned stimulus. Thus, the difference between self-adaptation and conditioning is that adaptation requires only one stimulus whereas conditioning requires two stimuli for the association. In other words, the animal is able to establish new association between the novel stimulus and response.

\section{ASSOCIATIVE LEARNING}

This type of learning is often called "associative learning" since it establishes association between stimuli and responses. In the above classical conditioned eye-blink reflex, the US is the air-puff and the CS is the tone. They are paired together to establish the subsequent association between CS and CR. That is, presentation of tone will elicit an eye-blink response after repeated pairing whereas such association did not exist prior to the conditioning experiment (i.e., presentation of tone would not elicit an eye-blink prior to the training phase).

\section{TRANSFER OF ASSOCIATION FROM INNATE STIMULUS TO A NOVEL STIMULUS}

Fig. (1) illustrates the transfer of US to CS to establish the CS-CR stimulus-response function using a block diagram, and the corresponding simplified neural circuitry. In the neural circuitry, the synaptic efficacy (connection weight strength) for the CS-CR pair is zero before training whereas the synaptic weight for CS-CR pair is increased subsequent to training (repeated associative conditioning). The strength- ening of the connection weight is induced by the activation of the US-UR pair, thus transferring the original US-UR stimulus-response function to the novel CS-CR stimulus-response function.

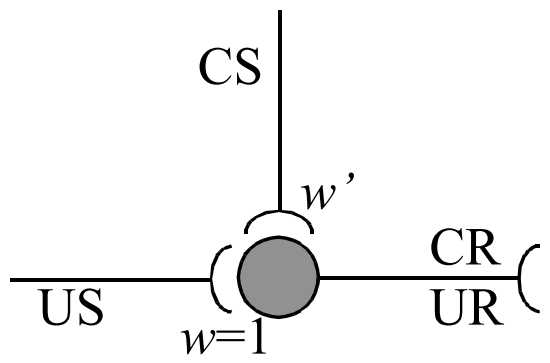

Fig. (1). Schematic diagram of a simplified neural circuitry for transferring the unconditioned stimulus (US) to conditioned stimulus (CS) via the modification of connection weight, $w^{\prime}$, at the CSCR synapse induced by the US-UR synapse (fixed connection weight, $w=1$ )

\section{MODIFIABLE REFLEX}

This illustrates that reflexes are modifiable rather than fixed or strictly hardwired. The original (innate) stimulusresponse I/O function can be altered such that the original stimulus does not need to be presented to elicit a response. This transfer of stimulus from the original innate form to a novel stimulus can be very different in quality (i.e., from air pressure to sound frequency in the above example). The transferred stimulus can also be different in energy form, e.g. from air-puff to light (from mechanical energy to photo energy) if the conditioning is paired between air-puff and lightonset stimuli, in which the animal will subsequently blink whenever the light is turned on.

\section{POSITIVE AND NEGATIVE REINFORCEMENT}

Central to the neural mechanism of conditioning is the reinforcement signal in which the response is reinforced. There are two major classes of reinforcement - positive reinforcement and negative reinforcement.

Reward is considered as positive reinforcement whereas punishment is considered as negative reinforcement. Positive reinforcement often leads to affiliative (seeking/attractive) behaviors whereas negative reinforcement often leads to avoidance (repulsive) behaviors.

\section{REINFORCEMENT LEARNING}

Because of these characteristic responses, conditioning paradigm is often called behavioral shaping. The end-goal to be shaped is either seeking behavior (for positive reinforcement) or avoidance behavior (for negative reinforcement). In either case, the neural mechanism in establishing such stimulus-response function is associative learning (or conditioning). Thus, this type of reinforced associative learning is called "reinforcement learning" in neural network community whereas it is called "conditioning" in psychology.

\section{REINFORCER}

The transfer of US to CS stimuli to the CS-CR stimulusresponse function in the classical conditioning paradigm is shaped by the reinforcement signal called the "reinforcer". 
Pairing with a positive reinforcer (reward) tends to promote affiliation whereas pairing with negative reinforcer (punishment) tends to promote avoidance.

\section{GOAL-DIRECTED LEARNING}

Reinforcement learning requires a reinforcer to establish the goal-directed behavior. The reinforcer is often the US, but the reinforcer can be derived from an alternate source too, as explained below. The direction of the end-goal is dependent on whether the reinforcer is a positive or negative reinforcement. The behavioral outcome of the animal (or autonomous being) in this conditioning paradigm can be directed toward seeking-behavior or avoidance-behavior. Thus, this type of reinforcement learning is sometimes called "goaldirected learning" [11-13].

Although many other neural network models were implemented with conditioning as the learning paradigm to solve problems (such as [14]), those models often are not addressing or solving problems related to emotions; whereas the model introduced in this paper focuses on the basic principles of operation for establishing emotional context in sensation.

\section{NEURAL NETWORK IMPLEMENTATION}

Theoretical foundation of neural network has been established extensively to explain many high-level cognitive functions, such as learning and pattern recognition [15]. In brief, the brain of an animal is essentially composed of many networks of neurons. By definition, neural network is essentially a set of interconnecting neurons (neural elements). The function of a neural net is to process information collectively by its neurons.

\section{NETWORK CHARACTERISTICS}

One of the characteristics of a neural net is that the overall I/O function processed by a neural net is performed by the collective properties of many subsets of neurons rather than strictly by each individual neuron. Although each neuron does have its individual $\mathrm{I} / \mathrm{O}$ function to be processed, the $\mathrm{I} / \mathrm{O}$ function of a network is often very different from the individual I/O function. In fact, the resulting properties exhibited by a neural network's processing may not be found in its component (i.e., the neurons).

\section{EMERGENT PROPERTY}

This property exhibited by a neural network that cannot be found in its component neurons is often called "emergent property". Examples of emergent properties of neural networks are learning and pattern recognition. Since most of the neural I/O functions are nonlinear, the overall I/O function of the network cannot be described by the linear sum of the I/O function of each neuron; thus this allows the emergence of processing properties from the network that are not found in individual neurons.

\section{MANY-TO-MANY MAPPING IN NEURONS}

For neurons in a network with multiple connections, the $\mathrm{I} / \mathrm{O}$ function of Eq. 9 is not merely a one-to-one mapping, but a many-to-many mapping mathematically. In other words, the mapping is not merely scalar, but vector. This many-tomany mapping for a network can be represented by a vector or matrix:

$$
\vec{Y}=c \cdot f(\vec{X})
$$

\section{CONNECTION WEIGHTS}

One of the characteristics of neural networks is that the neurons are interconnected with a connection weight, $w$, such that the individual inputs are scaled by the connection weight. Furthermore, the connection weight, $w$, is modifiable such that it is adaptive over time, governed by a set of learning rules.

\section{SYNAPTIC STRENGTH}

Biologically, the connection weights correspond to the synaptic strengths of neurons. The synaptic strength can be positive in excitatory synapse, and negative in inhibitory synapse, and zero for a non-functioning synapse (or no connection between two neurons). The synaptic strength for biological neurons can also be modified, which is called synaptic plasticity in neurobiology.

\section{MULTIPLE-GAIN CONTROL SYSTEM}

This connection weight can also be considered as the "gain" function in feedback control systems. Thus, the connection weight is essentially the gain in an adaptive control system even though each input has its individual gain function rather than a single gain function in a typical adaptive feedback control system. This multiple-gain adaptive control system provides the essential mechanism for learning in neural network.

\section{NEURAL NETWORK}

The generalized Eq. 10 can be implemented as a function of time, $t$, specifically by including the connection weight gain matrix, $w(t)$, as follows:

$$
\vec{Y}(t)=f(\vec{W}(t), \vec{X}(t))
$$

or

$$
y_{j}(t)=f\left(w_{i j}(t), x_{i}(t)\right)
$$

where $x_{i}$ represents the $i^{\text {-th }}$ input of the neuron, $y_{j}$ represents the $j^{\text {-th }}$ output of the neuron, and $w_{i j}(t)$ the connection weight between $i^{\text {th }}$ input and $j^{\text {-th }}$ output of the neuron (see also Fig. (2)).

\section{WEIGHTED-SUM}

In most neural networks, a weighted-sum function is used such that the output of a neuron is given by:

$$
y_{j}(t)=f\left(\sum_{i=1}^{n} w_{i j}(t) x_{i}(t)\right)
$$

for a total of $n$ inputs.

Thus, the output of a neuron is the weighted-sum of its input, adjusted by the individual gain, $w_{i j}$ (or connection weight between the $i^{\text {th }}$ input and $j^{\text {-th }}$ output). 


\section{THRESHOLDING FUNCTION}

In most neural networks, the nonlinear I/O function, $f(\cdot)$, is a thresholding function, either implemented as a hardthreshold (step-function) or a soft-threshold (sigmoidal function). For hard-threshold, a step function is often used:

$y_{j}(t)= \begin{cases}y_{\max } & \text { if } \sum_{i=1}^{n}\left(w_{i j}(t) x_{i}(t)\right) \geq \theta \\ y_{\min } & \text { otherwise }\end{cases}$

where $\theta$ denotes the threshold, for a neuron with $n$ inputs, and $y_{\max }$ and $y_{\min }$ are the corresponding high and low values of output, respectively. For a soft-threshold, a sigmoidal function is often used:

$y_{j}(t)=\frac{1}{1+e^{-\sum_{i=1}^{n}\left(w_{i j}(t) x_{i}(t)\right)}}$

The use of the sigmoidal function allows the above I/O function differentiable in the minimization process mathematically.

These nonlinear functions essentially provide the threshold for activating the output given the weighted-sum of the inputs. In other words, individual gains are applied to each of the inputs, which are then summed together to produce the output set by the threshold.

\section{MULTI-GAIN ADAPTIVE CONTROL SYSTEM}

In order for a control system to be adaptive, the variable gains can be applied instead of using fixed gains. In other words, the connection weights can be adjustable. Furthermore, the connection weights are essentially the individual gain applied to each of the input of the system such that the input signals are biased (amplified or attenuated) by the gain or the connection weight. The difference is that there is individual gain for each of the input instead of the conventional single gain signal that applies to the entire system in most conventional control systems.

\section{MODIFIABLE MULTI-GAIN SYSTEM AND LEARN- ING}

Since the connection weights are modifiable (adaptable gains), this phenomenon of modifiable synaptic efficacy in biological neurons forms the biological substrate for learning and memory [16].

The rules for modification of these connection weights become the "learning rules" in neural networks, since by applying these rules to modify the connection weights, the neural net system as a whole can exhibit the emergent property of "learned behavior".

\section{HEBBIAN ASSOCIATIVE LEARNING RULE}

There are many learning rules commonly used in neural network [15], such as associative learning rule (Hebbian learning rule) [17], back-propagation learning rule [18], etc. The most relevant learning rule in this context is the associative Hebbian learning rule. Not only does this Hebbian rule form auto-association naturally, but also the association mechanism is most biologically plausible.

Hebb [17] in 1949 proposed that if the pre-synaptic and post-synaptic neurons are activated together, then the synaptic strength could be changed. This is essentially the associative learning rule where the input and output are correlated together to change the connection weight. Hebbian associative learning rule has been applied in numerous neural network systems and in neurobiology. We will summarize the Hebbian learning rule briefly below.

The Hebbian associative learning rule is given by:

$$
\Delta w_{i j}(t)=l \cdot y_{j}(t) \cdot x_{i}(t) \quad \forall i, j
$$

and

$l^{\prime}=l / \Delta t$

where $\Delta w_{i j}$ is the incremental weight change at time $t, l$ is the learning coefficient (corresponding to the scale-factor parameter for incremental weight change), and $l^{\prime}$ is the learning-rate.

The above equation satisfies the Hebbian rule because the connection weight changes only when the input, $x_{i}(t)$, and output, $y_{j}(t)$, are activated (i.e., non-zero). If either one is zero (i.e., if either input or output is not activated), no weight change occurs.

If the above functions are expressed in discrete time-step, $\Delta t$, the weight at the next time step, $t+\Delta t$, is given by:

$w_{i j}(t+\Delta t)=w_{i j}(t)+\Delta w_{i j}(t) \quad \forall i, j$

Alternatively, in engineering perspective, the connection weights essentially provide the adjustable/adaptable gain changes needed for associative learning such that it correlates the specific input with the corresponding output by the biases provided by the gains. Thus, Eqs. $14-17$ form the set of equations for Hebbian associative learning for individual neurons.

\section{NETWORK LAYERS}

Neurons can be interconnected together to form a network. Without loss of generality, a network can be considered as neurons forming layers, from input layers to output layers via some intermediate layers. Thus, the description of the $\mathrm{I} / \mathrm{O}$ function of a generic neuron at any given $k^{\text {-th }}$ layer is given by:

$$
\vec{Y}^{k}(t)=f\left(\vec{W}^{k}(t) \cdot \vec{X}^{k}(t)\right)
$$

or

$y_{j}^{k}(t)=f\left(\sum_{i=1}^{n} w_{i j}^{k}(t) x_{i}^{k}(t)\right)$

(see Fig. (2)), and the associative weight-change learning rule is given by:

$$
\begin{aligned}
& \Delta w_{i j}^{k}(t)=l \cdot y_{j}^{k}(t) \cdot x_{i}^{k}(t) \quad \forall i, j, k \\
& w_{i j}^{k}(t+\Delta t)=w_{i j}^{k}(t)+\Delta w_{i j}^{k}(t) \quad \forall i, j, k
\end{aligned}
$$


Thus, Eqs. 18 - 19 form the set of equations for Hebbian associative learning for individual neurons at the $k^{\text {th }}$ layer in a network.

Note that the above equations specify the I/O function of a neuron at the $k^{\text {th }}$ layer from its $i^{\text {-th }}$ input to its $j^{\text {th }}$ output only (without explicitly specifying which layer the input comes from or which layer the output goes to). Different connectivity will provide different network architecture.

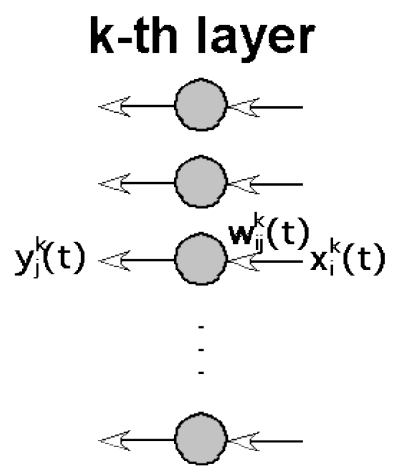

Fig. (2). Schematic diagram showing the neural input and output at the $k^{\text {th }}$ layer.

\section{NETWORK ARCHITECTURE}

In general, the most extensive network is a fully connected network with all-to-all connections. Alternatively, a network can form layers, with either feedforward connections or feedback connections or both. Furthermore, connections can form layers not just to the adjacent layer, but also bypass the adjacent layers. Thus, different network architecture exists that would provide different properties of processing. In this paper, we provide a generalized theoretical foundation for derivation of emotional context without restricting the network architecture to any specific type.

\section{CONNECTIONS BETWEEN LAYERS}

Taking the specific connectivity into account, we will use the superscript notation of $m k$ to denote the connection from $m^{\text {th }}$ to $k^{\text {th }}$ layer, and $k n$ to denote the connection from $k^{-}$ th to $n^{\text {-th }}$ layer. The description of $\mathrm{I} / \mathrm{O}$ function of a neuron at the $k^{\text {th }}$ layer connecting from $m^{\text {th }}$ layer to $n^{\text {th }}$ layer is given by:

$$
\vec{Y}^{k n}(t)=f\left(\vec{W}^{m k}(t) \cdot \vec{X}^{m k}(t)\right)
$$

or expanding it:

$$
y_{j}^{k n}(t)=f\left(\sum_{i} w_{i j}^{m k}(t) x_{i}^{m k}(t)\right)
$$

(see also Fig. (3)) and the corresponding associative weightchange learning rule is given by:

$$
\begin{aligned}
& \Delta w_{i j}^{m k}(t)=l \cdot y_{j}^{k n}(t) \cdot x_{i}^{m k}(t) \quad \forall i, j, k, m, n \\
& w_{i j}^{m k}(t+\Delta t)=w_{i j}^{m k}(t)+\Delta w_{i j}^{m k}(t) \quad \forall i, j, k, m, n
\end{aligned}
$$

Note that since input and output are relative as far as any neuron is concerned, so the input from the $m^{\text {-th }}$ to $k^{\text {th }}$ layer for a neuron at the $k^{- \text {th }}$ layer is the output from $m^{\text {th }}$ to $k^{\text {th }}$ layer for a neuron at the $m^{\text {-th }}$ layer (see also Fig. (3)):

$x_{i}^{m k}(t)=y_{j}^{m k}(t) \quad \forall m k$ pair

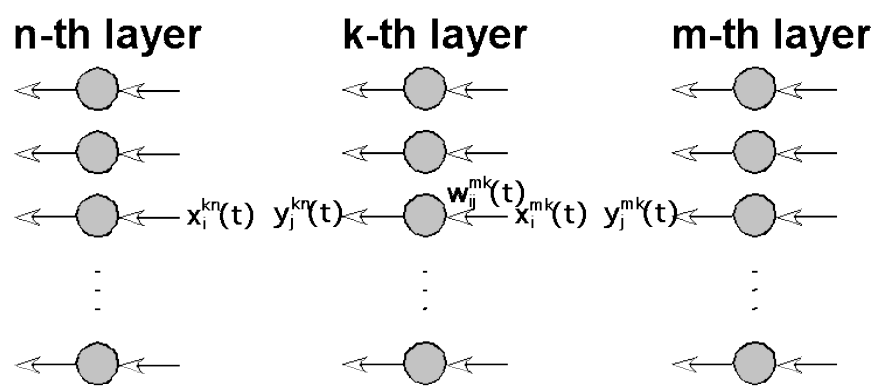

Fig. (3). Schematic diagram showing the network connectivity at the $k^{\text {th }}$ layer, connecting from the $m^{\text {-th }}$ layer to the $n^{\text {-th }}$ layer.

\section{NORMALIZATION OF CONNECTION WEIGHTS}

Since the connection weights can increase indefinitely with each incremental time, $\Delta t$, normalization of these weights can resolve this dilemma. One of the normalization schemes is given by:

$$
{w_{i j}^{\prime}}^{\prime k}(t)=\frac{w_{i j}^{k}(t)}{\left|\sum_{i j k} w_{i j}^{k}(t)\right|} \quad \forall i, j, k
$$

where $w_{i j}^{\prime k}(t)$ is the normalized weight to be substituted in the above equations. (For simplicity, and without loss of generality, we will use the notation for neurons at the $k^{\text {th }}$ layer without specifying the notation between layers from here on.)

\section{CROSS-CORRELATION FUNCTION AND ASSOCIA- TIVE HEBBIAN LEARNING}

The associative learning rule of Eq. 19 or 21, provides a mechanism for correlating the input with the output. In fact, with a time-delayed network architecture, it can be proved that a time-delayed associative Hebbian learning network essentially performs a mathematical cross-correlation function computation between input and output streams $[19,20]$.

Given that the significance of associative learning is performing a cross-correlation function, associative learning can be interpreted as correlating the sensory inputs with its own output actions to establish some significance (contextual meaning) of the input-output pairs. When multiple inputs and multiple outputs are included in this cross-correlation with a nonlinear, multi-layered neural network architecture, the emergent I/O relationship of the network can become contextual relative to the environmental context, i.e., sensory input with respect to the system's output.

\section{ASSOCIATION BY CROSS-CORRELATION}

The exact correlated context (extracted or abstracted by the network) depends very much on the neural net architecture and interconnectivity, such as feedback or feedback networks, which we will discuss further later. We will continue our discussion with how this mechanism for association (or correlation) can be used to guide (or shape) the behavior, i.e., reinforce the animal (or autonomous robot) in such a way 
that the system will either seek or avoid the associated stimuli with its actions.

Because association between the input and output alone does not necessarily provide the clue needed for behavioral shaping of either seeking-behavior or avoidance-behavior, additional stimulus (input) is required. This addition signal is the reinforcement signal, i.e., positive reinforcement for reward and negative reinforcement for punishment.

\section{ASSOCIATIVE REINFORCEMENT LEARNING}

In order for an animal (or autonomous robot) to seek or avoid certain stimuli for behavioral guidance, an additional signal, $z(t)$, can be used as a reinforcer to change the connection weight in the associative learning rule of Eq. 19. The associative reinforcement-learning rule that includes a reinforcer is given by:

$\Delta w_{i j}^{k}(t)=l \cdot z(t) \cdot y_{j}^{k}(t) \cdot x_{i}^{k}(t) \quad \forall i, j, k$

where $z(t)$ denotes the reinforcement signal at time $t$ associated with input $x_{i}^{k}(t)$.

\section{THREE-WISE CORRELATION}

Note that the above learning rule for weight change is essentially a three-wise correlation, correlating between the input, output and reinforcer. In comparison, the classical Hebbian learning rule is a two-wise cross-correlation between input and output.

\section{SENSORY INPUT AS REINFORCER}

Note also that, in classical conditioning, the reinforcer, $z(t)$, is derived from one of the sensory inputs, $x_{i}^{k}(t)$. In other words, there is special significance in one of these inputs as far as the processing is concerned. In this case, one of the inputs is treated as the reinforcer to shape the behavior. Thus, this special input has profound implication in altering the course of action for the network. (We will discuss how an alternate reinforcer can be derived from other sources later.)

\section{REINFORCER AS GAIN CONTROL}

Note that the reinforcer, $z(t)$, is essentially another "gain control" signal used for auto-adaptation (modification of connection weight). In other words, the weights are changed depending on not only the learning coefficient, $l$, but also the size of the reinforcement (i.e., the gain), in addition to the activation of both input and output of that neuron. The larger the reinforcer signal, the bigger the gain is applied to the weight change.

\section{SIGNIFICANCE OF REINFORCER AND GAIN}

If the reinforcement signal $z(t)$ is positive, it will lead to an increase in connection weight. This means the output action is more likely to be positive as a result, which means acting toward the stimuli, i.e., reinforced positively. The animal (or autonomous robot) will be more likely to move toward the stimuli as a result. The gain for the weight change is positive in this case.

Conversely, if the reinforcement signal is negative, it will lead to a decrease in connection weight, resulting in rein- forced negatively, leading the animal to be more likely to avoid taking actions toward the stimuli. Thus, the gain for the weight change is negative in this case.

\section{NEUTRAL ENVIRONMENT}

If the reinforcement signal $z(t)$ is zero, it means the learning trial is not reinforced. In such case, from Eq. 24, $\Delta w_{i j}^{k}(t)=0$, no learning occurs. This means the system remains constant as is, without changing when it is not reinforced. In other words, since the system is neither reinforced positively or negatively, the environment is considered as neutral, it will remain neutral in its learning.

\section{LEARNING FROM LEARNING}

Although it may seem intuitive to assume some baseline learning to occur in a neutral environment (when the reinforcer signal $z(t)$ is zero), the fact is, if learning were to occur (in our definition), it has to bias the system toward or away from the end-target, which would imply either positively or negatively reinforced. Nonetheless, learning about neutrality can occur at a higher-level processing.

Learning about the neutrality of the setting can be obtained from a higher-level learning, which can be derived from another super-set network on top of the current network such that the inputs of this super-set network are derived from the current front-end network (rather than derived from the sensory input of the environment).

\section{SUPER-SET NETWORK AND PRE-PROCESSOR}

In many ways, abstraction of learning can be accomplished by generalizing this framework, forming networks of networks to process higher-level abstraction of the output from the pre-processor networks. This abstraction of processed output by super-set networks of networks essentially forms the basis of emotional processing to guide its learning or behavioral path.

\section{SUPERVISED REINFORCEMET LEARNING}

The reinforcer, $z(t)$, can be delivered by an external source, such as a "teacher", in which case, this type of reinforcement corresponds to classical conditioning. In neural networks, this type of learning is called "supervised learning".

\section{UNSUPERVISED REINFORCEMENT LEARNING}

If the reinforcer is generated by the action of the system (the animal or autonomous robot) itself, then this type of reinforcement corresponds to operant conditioning in psychology. The animal, thus, learn without a "teacher", which can be considered as self-learning or auto-associative learning. This class of learning is also called "unsupervised learning".

\section{GOAL-DIRECTED LEARNING}

Independent of whether the reinforcement signal is delivered by an external "teacher" or not, this class of learning is often considered as "goal-directed" reinforcement learning [11-13] since the reinforcer provides the learning cue in the 
direction of the reinforced behavior, i.e., positively or negatively reinforced.

\section{SOURCE OF REINFORCEMENT SIGNAL}

The reinforcement signal, $z(t)$, in Eq. 24 can come from many different sources. It can come from the sensory input, such as food for animals or battery recharging signal for autonomous robots for positive reinforcers, and pain signal for negative reinforcer. These basic reinforcers are often innate signals that are hardwired into the system's circuitry for basic survival. These signals are also referred to as unconditioned stimulus (US) signals. (The mechanism for the formation of these innate responses will be addressed later in this paper after the mechanisms for the transfer of internal reinforcements within the environmental context are introduced with respect to the meta-system).

\section{POSITIVE AND NEGATIVE REINFORCERS}

Since the reinforcer (such as food or pain) is derived from one of the sensory inputs in the first-layer, a positive reinforcer can be represented by:

$z(t)=x_{h}^{1}(t)$

if the reinforcer is the $h^{\text {th }}$ input of the system representing the US signal, and a negative reinforcer, $z^{\prime}(t)$, can be represented by:

$z^{\prime}(t)=-x_{h}^{1}(t)$

Eqs. 25 and 26 can be combined to a single equation for both positive and negative reinforcements. The reinforcer is given by a generic form:

$z^{\prime}(t)=r \cdot x_{h}^{1}(t)$

where a reinforcement gain coefficient, $r$, is used to encapsulate either positive or negative reinforcement such that $r>0$ represents positive reinforcement, and $r<0$ represents negative reinforcement.

If the reinforcer is exactly the same as the $h^{\text {th }}$ input of the neuron without amplification or attenuation, then $r=1$ for positive reinforcement, and $r=-1$ for negative reinforcement.

\section{ASSOCIATIVE REINFORCMENT LEARNING RULE}

Applying this generalized reinforcer gain, $r$, to Eq. 24, the learning rule for weight-change equation becomes:

$\Delta w_{i j}^{k}(t)=l \cdot r \cdot x_{h}^{1}(t) \cdot y_{j}^{k}(t) \cdot x_{i}^{k}(t) \quad \forall i, j, k, h$

where the incremental size and direction of connection weight-change are dependent on the learning coefficient, $l$, and reinforcer gain, $r$.

\section{TRANSFER OF REINFORCER}

Other reinforcement signals can be derived from other sensory inputs, such as the light signal paired with the innate reinforcer in classical or operant conditioning. These signals are often referred to as conditioned stimulus (CS), in which a secondary sensory signal is used to derive as the reinforcement signal for associative learning.
The transfer from the innate reinforcer (US), $x_{h}^{1}(t)$, (at the $h^{\text {th }}$ input in the first-layer) to the acquired reinforcer (CS), $x_{m}^{1}(t)$, (at the $m^{\text {th }}$ input in the first-layer) can be established by associative correlation using the similar associative learning mechanism.

Once conditioning is established, the new CS reinforcer, $x_{m}^{1}(t)$, becomes the substituted reinforcer, giving the new reinforcement learning equation for CS signal:

$$
\Delta w_{i j}^{k}(t)=l \cdot r \cdot x_{m}^{1}(t) \cdot y_{j}^{k}(t) \cdot x_{i}^{k}(t) \quad \forall i, j, k, m
$$

\section{INTERNAL REINFORCER}

By the same token, applying similar associative learning paradigm for transfer of reinforcer signal from one input to another, internal neural signals can be used as reinforcement signals. In other words, the reinforcer does not need to be originated from external sensory signals (first-layer input); input in the internal-layer can be used as reinforcer.

Thus, the original innate reinforcer, $x_{h}^{1}(t)$, in the $h^{\text {th }}$ input at the first-layer can be substituted (or replaced) by an acquired reinforcer, $x_{m}^{k}(t)$, in the $m^{\text {th }}$ input at the $k^{\text {th }}$ layer. Eq. 29 can now be rewritten as:

$$
\Delta w_{i j}^{k}(t)=l \cdot r \cdot x_{m}^{k}(t) \cdot y_{j}^{k}(t) \cdot x_{i}^{k}(t) \quad \forall i, j, k, m
$$

In other words, the system is able to derive its own reinforcer internally for associative reinforcement learning rather than deriving from the external sensory source.

\section{VIRTUAL REINFORCER}

This internally derived reinforcer can be considered as a virtual reinforcer (virtual reward or virtual punishment). The virtual reinforcer can become a powerful mechanism for selfguided learning in motivating the animal (or autonomous robot) to seek or avoid certain environment conditions represented (encoded) by the set of sensory stimuli without external reinforcer. In other words, contextual abstraction of the sensory stimuli can be derived by such internal associative representation for a given context.

\section{REINFORCER AS AN IMPLICIT GUIDE}

The above analysis illustrates that the reinforcer signal plays a crucial role in determining the direction of the endgoal for a goal-directed behavior. In many ways, the reinforcement signal can be considered as the "switch" to guide the behavior toward or away from the desired goal. Although the "desired goal" may or may not be defined (or known) as far as the animal is concerned, we use the term desired goal in the theoretical sense to indicate what the system will eventually arrived at, given the conditioning paradigm.

\section{CONDITIONED FEEDBACK REINFORCEMENT}

The reinforcement-guiding signal can be derived from either feedforward or feedback signal. Which signal it uses depends on whether the system's output (the animal's response) is incorporated as the feedback reinforcement. If the action of the animal results in an alteration of the reinforce- 
ment signal, then it is a form of "feedback reinforcement" automatically.

For example, if an animal moves toward the food (reward) as a result of the conditioning, it increases the positive reinforcement signal by its motor response. This would strengthen the synaptic weight in the neural circuitry, resulting in a further increase in subsequent response size.

This is essentially self-learning, associative learning without a "teacher", unsupervised learning or operant conditioning, where the behavior is shaped by self-exploration of the animal instead of being guided by an outsider.

\section{CONDITIONED FEEDFORWARD REINFORCEMENT}

If the reinforcement signal is a feedforward signal in which the response of the animal does not affect the presentation of the reward or punishment, it is a form of "feedforward reinforcement". For example, the Pavlovian conditioning is an example in which Pavlov [21, 22] presented the food reward independent of the behavioral response of the dog.

This type of feedforward reinforcement corresponds to classical conditioning (or Pavlovian conditioning), learning with a "teacher", or supervised learning. In both case, the behavior is shaped by the reinforcement signal whether it is a feedforward or a feedback signal.

\section{DERIVED REINFORCER}

When a reinforcement system receives its reinforcer signal from an external source, such as food reward, then it is a feedforward system in which the reinforcer is directing the system's adaptation without relying on any internal feedback signal for adjustment. But when the system is deriving an alternate reinforcement signal other than the original reinforcer, such as in a conditioning paradigm, then it becomes a feedback system since the reinforcer is no longer coming from an independent external source, but deriving from a dependent internal source.

\section{CONDITIONED FEAR AS AN EXAMPLE}

Conditioned fear is an example where a reinforcer can be substituted by an alternate source. The original fear response is triggered by a noxious stimulus. But when it is paired with another stimulus, the alternate signal acts as a reinforcer triggering the response. For instance, when a shock is paired with a tone signal, the original reinforcer (US shock) is no longer needed to elicit the fearful response while the alternate reinforcer (CS tone) is sufficient to elicit the conditioned fear response.

\section{CONDITIONING BY BENIGN REINFORCER}

Subsequent to this fear conditioning, another CS (such as pairing the tone with yellow warning light) could be used to establish an alternate stimulus-response function using the secondary reinforcer (tone). Thus, the transfer of the reinforcer from the original source (noxious stimulus) to an alternate source (non-noxious stimulus) forms a feedback loop.

Note that, in this case, even though the reinforcer is no longer noxious (i.e., benign or neutral), the $\mathrm{CR}$ response is still avoidance behavior rather than habituation (or extinc- tion) because the response is associating with the original previous noxious stimulus (cascaded from the transfer of reinforcers).

\section{ENVIRONMENT AS THE FEEDBACK LOOP}

In most circumstances, the output action is a feedforward action in which any errors in the output are not directly (or explicitly) fed back into the system for correction as far as the learning rule for the neural circuitry is concerned. But that is not to say that the system does not receive any feedback from its action.

In reality, because of reinforcement, a change in course of action occurs. As a result, the subsequent behavioral action alters the environment (such as moving away from the aversive stimulus) in such a way that it also changes the reinforcement intensity (diminishing the intensity of reinforcement signal when it moves away from that source). Thus, a feedback loop is still maintained even though the loop includes the external environment, not just the autonomous system itself.

\section{COMBINING AUTONOMOUS BEING AND ITS EN- VIRONMENT AS A META-SYSTEM}

The above discussion leads to the expansion of the scope included in the components of a system. Although most of the time, the autonomous robot (or animal) is considered as a standalone system with its own self-actuating and selfadaptive components, we may include the environment in which it operates as a meta-system.

Because the animal (or autonomous robot) is no longer operating in isolation independent of the environment, the environment becomes an integral part of the meta-system it operates in. Every action it takes may have an impact on the environment, thus the resulting action provides a feedback to the organism indirectly through the alteration of the environment its exposes to.

Thus, the autonomous system cannot be viewed in isolation without the environment considered as part of the metasystem. In other words, the context of the sensory signals is only meaningful in relation to how they affect the organism, and is often meaningless without the organism in place.

\section{ENVIRONMENTAL CONTEXT IN EMOTION FOR- MATION}

By the same token, the emotional context of the sensation is only meaningful when the environment is present; it is meaningless without the environment. Thus, it is essential to include the environment as part of the meta-system when autonomous control and emotional context are considered together.

\section{SELF-EXPLORATION AS PARAMETER SPACE SEARCH}

For feedback reinforcement, self-learning can be acquired by exploration, i.e., variation (randomization) of the response output. Small variations in the motor output allow the animal (or autonomous robot) to explore different parameter space in search for the final I/O function. Thus, probabilistic stimulus- 
response I/O function (such as Eqs. 5 - 8) can enable the system to "explore" the parameter space, whereas deterministic I/O function (such as Eqs. 3 - 4) often does not.

\section{SOLUTION WITHOUT A PRIORI KNOWLEDGE}

The exploration of the parameter space can be done by an animal itself (without a "teacher") to establish the final probabilistic I/O function by incorporating a feedback reinforcement signal together with a stochastic I/O function without any presumed knowledge of the outcome of the system.

In contrast, exploration of the parameter space for deterministic I/O functions often requires feedforward reinforcement signal provided by the "teacher" who predicts (projects) what the final outcome (desired goal) would be, with presumed knowledge of the outcome of the system.

\section{ROLE OF PROBABILISTIC FUNCTION IN SELF- EXPLORATION}

The mathematical mechanism for self-exploration lies in the probabilistic I/O function. Without the variations in output, the animal (or autonomous robot) will always repeat the same action unless its action is altered by environmental perturbations or by an external "teacher".

Environmental perturbations do occur in the real world due to unforeseen circumstances, or failure of moving parts. Thus variability in sampling the parameter space can occur in deterministic system, but it is incidental rather than intrinsic to the system's operation.

This is consistent with the fact that most biological systems are variable in their output action rather than rigidly stereotypical or identical. In fact, variability in output actions is the hallmark of animals whereas precise output is the hallmark for machine actions.

\section{ESTABLISHING EMOTIONAL CONTEXT INTER- NALLY BY REINFORCEMENT LEARNING}

The next phase in deriving the emotional context in sensation is to establish the neural mechanisms for the abstraction of emotional feel. Conditioning and associative reinforcement learning is well suited for the mechanism in establishing emotional context associated with the sensory stimulus.

\section{INTERNAL AFFILIATION OR AVOIDANCE}

Although traditionally, positive or negative reinforcement leads to behavioral output, i.e., motor response of either seeking or avoidance responses, similar responses can be established internally within the neural system rather than externally interacting with the external world. The internal representation of the affiliation or avoidance behavior prior to the motor output can be considered as the emotional representation of such behavior.

\section{MECHANISMS FOR TRANSFER OF DERIVED RE- INFORCER}

As illustrated before, when a reflex response is conditioned to another stimulus other than the original stimulus, the transfer of one stimulus to another can be established.
The successive transfer of other stimuli that are associated with the original stimulus can form the framework for establishing the context in which the original stimulus-response function is operating. In other words, the original sensory input is no longer interpreted (processed) in isolation; rather it is processed in reference to the other associated stimuli.

\section{NEURAL CORRELATE OF INTERNAL REWARD CENTER}

Nucleus accumbens is the brain structure in the mesolimbic system that is well known for its behavioral reinforcement property in mammals [23]. Activation of the nucleus accumbens often produces highly reinforced behavior, especially in reward activation. It is also known that nucleus accumbens is activated by different types of reward signals, including water [24], food [25], dopaminergic drugs [26, 27] such as cocaine, and even visual stimuli such as beautiful faces [28]. Cocaine is known as a powerful internal reinforcer in behavioral activation [29]. Thus, internal loci of reinforcer exist in animals.

\section{FEEDBACK GAIN BIAS IN ESTABLISHING EMO- TIONAL CONTEXT}

The above discussion shows that associative reinforcement learning can be one of the mechanisms for establishing context of a sensation. This reinforcement paradigm is essentially a feedback control system whereby the sensory stimuli are integrated into the neural processing, not only as sensory inputs per se, but also as internal multiple-gain feedback control signals. The gain can be positive or negative, which can be used to automatically set "biases" to the system in such a way that certain sensory inputs are amplified (or attenuated).

\section{ESTABLISHING EMOTIONAL SIGNIFICANCE BY INCREASING THE GAIN}

The amplification of these specific sets of inputs signifies the "importance" of the signal in establishing association, i.e., correlation among these input signals. That is to say, the signals are self-selected in the processing such that they form special "significance" in determining the final output. Conceptually, this process establishes the "context" from the environment by integrating the sensory signals that have significant importance for determining its output.

\section{SELF-SELECTIVE BIAS IN EMOTION FORMATION}

This "self-selective" process for biasing the system to put more importance of the selected signal to produce its output is relied on the feedback information derived from the sensory signals themselves. This also assumes that the initial starting point is relied on the existence of a presumed innate reinforcer that sets the direction of behavioral motivation, i.e., positive reinforcer (such as food) will increase the probability of motor activation for affiliation (seeking-behavior) while negative reinforcer (such as pain) will tend to increase the likelihood of motor activation for avoidance motor output. (We will show in subsequent sections how this innate reinforcer can be established.)

INNATE AND VIRTUAL REINFORCER AS INTERNAL TEACHER 
The innate reinforcer essentially serves as the internal "teacher" that guides the autonomous system's behavior. Subsequent acquisition of other signals as the reinforcer can be established by feedback reinforcement by correlating the initial innate reinforcer with other potential candidate reinforcers. The new reinforcer established internally can be considered as the virtual reinforcer, as discussed earlier. This transfer of one reinforcer to another reinforcer can be selfpropagating using the conditioning paradigm.

\section{MECHANISMS FOR ESTABLISHING INNATE REIN- FORCER}

Given that the innate reinforcer can initiate the subsequent conditionings, the question may be raised to address how this initial innate reinforcer is established in the first place. In biology, innate properties often refer to in-born, genetically programmed properties. In engineering, these properties are often hardwired (or pre-programmed). Then the question becomes how genetic programming is established about without a designer or a programmer who may have a priori knowledge of the system or its desired outcome.

\section{ROLE OF FEEDFORWARD IN EVOLUTION}

One of the possible solutions to this problem of solving a problem without even knowing what the problem is lies in feedforward control. When a system uses a feedforward control for its operation, it does not require feedback to determine whether its actions are appropriate or not. It simply produces its output based on the feedforward signal independent of the outcome.

\section{FEEDFORWARD AS A PREDICTION}

This feedforward control often presumes a (wild guess) prediction of its output (even though that presumption may or may not be correct in actuality). Because of this presumption (predictive property) in feedforward control, it has the advantage of producing an action that may have a "chance" for success.

Metaphorically speaking, feedforward control allows the autonomous system to take chances at finding a solution even though it may be a "shotgun approach", but that is precisely the principle underlying the process of evolution.

\section{TRIAL-AND-ERROR APPROACH}

As in evolution, feedforward is used as the initial mechanism in the trial-and-error process to explore the parameter space. "Survival of the fittest" is the second step in the evolutionary process that is feedback in nature. That is, it reinforces (keeps) the trials that work, and minimizes (eliminates) trials that don't work. Without the feedforward mechanism, evolution would not proceed.

By the same token, using the feedforward evolutionary process, by trial-and-error, the innate reinforcer can be established.

\section{RANDOMIZATION IN PARAMETER SPACE SEARCH}

Central to this principle of feedforward evolutionary process is the trial-and-error exploratory process in sampling the parameter space. This trial-and-error process relies on the variations of the output.

The variations can come from many sources, both internal and external. External source may include perturbations to the system, such as mutation by radiation in genetics, or perturbations from the environment in autonomous robots. The autonomous system usually does not have much influence or control over these unforeseen external perturbation sources.

\section{ROLE OF INTRINSIC VARIATIONS}

In contrast to external perturbations that are beyond the control of the system, internal variations of its components can become part of the intrinsic properties of the autonomous system. Most often, these internal variations may take the form of probabilistic function or internal noise. The probabilistic output can provide the variations needed in the trial-anderror process for feedforward signal production. Thus, using the evolutionary approach, the innate reinforcer can be established when it is followed by the "fitness test" for survivalof-the-fittest feedback.

\section{FITNESS TEST FOR SURVIVAL}

Using a feedforward control function for exploratory solution, together with feedback control for fitness test, many of the innate properties found in animals or autonomous system can be formed by successive iterations. It is analogous to the principles used by "artificial life" or "genetic algorithm" [30] to explore the parameter space to find solutions to complex problems by random mutation and recombinant of subsolution space.

\section{ESTABLISHING INNATE REINFORCER WITHOUT A PRIORI KNOWLEDGE}

Using these feedforward and feedback computational mechanisms, the aforementioned innate reflexes and innate reinforcers can be established without any assumptions in the design (or a priori knowledge) of the appropriateness of the outcome of such system. No arbitrary artificial retrospective assignment of the roles/functions of these reflexes or reinforcers (i.e., what reflexes or reinforcers are used for) is needed. Their physiological (and psychological) functional roles are merely emergent properties of the system after extensive computational iterations.

\section{ESTABLISHING EMOTIONAL CONTEXT WITHOUT A PRIORI ASSUMPTIONS}

Similarly, the emotional context in sensation for an autonomous system can now be derived from first principles without any a priori knowledge or assumptions about what emotions are for. That is, it becomes an emergent property of the autonomous system when it goes through the feedforward and feedback cycles of iterations, consolidating (and reinforcing) "relevant" signals by the self-selective process for adjusting (adapting) the internal gains (connection weights and reinforcers) of the system integrating the input and output for establishing its probabilistic many-to-many I/O mapping functions. 


\section{EMOTION AS AN EMERGENT PROPERTY}

Thus, emotional sensation is an emergent property rather than a retrospective property that fits an artificial construct in psychology. Furthermore, emotions in autonomous robots are not necessarily pre-programmed or add-on entities introduced into the robot, but rather they are intrinsic parts of the sensory parameter space integrated with its internal processing for the production of appropriate output actions.

\section{CONDITIONED FEAR AS A CONTEXTUAL EMO-} TIONAL RESPONSE EXAMPLE

The conditioned reflex forms the background theoretical basis for the transfer of emotional responses from one type of stimulus to another. Fear conditioning [31], as illustrated before, is a classical example in neurophysiology [32] whereby the original stimulus that elicits the fear response is transferred to another stimulus, such as light-onset or toneonset when they are paired in the stimulus presentation to produce the conditioned fear reflex.

More complex response other than reflex can be invoked by conditioned fear to produce the contextual emotional response. It can be used to illustrate how environmental context can be incorporated into the emotional response that transfers from an innate response to a conditioned response.

\section{INTRINSIC FEAR}

Fear is one of the innate emotions experienced in animals to protect themselves from predation and other potential danger that may lead to death or self-destruction. Intrinsic fear is the in-born fear that is genetically programmed in animals when encountered with predators or height (fear of falling), for example.

For instance, when an animal approaches a cave and then discovered a bear in the cave, then subsequent to this pairing, the animal will be conditioned to be fearful in approaching a cave. Thus, the cave becomes the environment context of this fearful experience.

\section{SENSORY EXPERIENCE AND EMOTIONAL RE- SPONSE}

By the same token, an autonomous robot can acquire such fearful response when approaching a dark cave (sensory darkness) if it were conditioned by an aversive stimulus in the process using the same computational mechanisms. Thus, contextual meaning of the sensory inputs can be acquired from the environment in which the sensory experience is consolidated.

\section{BOOTSTRAPPING REFLEX}

Finally, we will address the mechanism for innate response formation, such as the establishment of reflex action. The above discussion focuses on the set of proposed mechanisms for producing the self-adaptability phenomena in autonomous systems such that emotional context can be established from the environment. Yet the derivation also depends on the existence of the presumed innate property (hardwired circuitry) within the system that it relies on for bootstrapping the subsequent associative reinforcement learning mechanisms. We will propose a mechanism whereby the innate characteristics (instinct) can be established.

\section{MECHANISMS FOR INNATE REFLEX FORMATION}

The mechanisms for innate response formation (such as reflex) depends on two theoretical principles:

1. the evolution mechanism of trial-and-error and survival-of-the-fittest test (i.e., initial feedforward exploration and subsequent feedback fitness-test in the meta-system) to sample the solution space; and

2. the consolidation of the circuitry (hardwiring) once the likely solution is found from the above evolutionary principle.

\section{PRINCIPLES OF EVOLUTION}

The evolutionary principle implicitly requires the inclusion of both autonomous agent and the environment as a meta-system for the evaluation of the fitness-test in survivability. The active exploration of solution space by using feedforward approach implies that the nonlinear function, $f(\cdot)$, in all of the above equations be probabilistic function rather than deterministic. The specific probabilistic function used for the autonomous system is an implementationspecific (species-specific) issue, which can be used to optimize the system's performance.

\section{ESTABLISHING INNATE RESPONSE BY FIXATING THE CIRCUITRY}

The consolidation of the internal neural circuitry (i.e., hardwiring) is opposite to the principle of learning (connection modifiability or synaptic plasticity) discussed above. In order to fixate the circuitry once the solution is approached, the learning-rate, $l^{\prime}$, can be decreased to zero.

\section{FREEZING THE LEARNING}

To freeze learning, the learning-rate needs be a function of time, $l^{\prime}(t)$, rather than a constant (pre-assigned as a parameter of the system as in most neural network learning equations). Thus, this learning-rate can self-adapt in much the same way as the aforementioned paradigm where the rate is high initially for exploration, and decreases as the system arrives at a stable solution. The fitness-test for survival is used as a criterion for evaluating the stability of the solution.

\section{CRITERION FOR FIXATION}

Without a priori knowledge of what the solution is, the autonomous system is still able to use the stability criterion for fixating the circuitry. The stability-test can be accomplished by evaluating the system's response over successive time-iterations. For instance, if the connection weights do not change significantly over multiple iterations of time-steps, the system can be considered as approaching a stable state.

\section{MOVING-AVERAGE AS A STABILITY MEASURE}

Many different stability criteria can be used; for illustrative purpose, we will provide one such stability criterion for evaluation using a moving-average function, $|\Delta \bar{w}|_{t}$, of the total weight-changes of the system at time, $t$ : 


$$
|\Delta \bar{w}|_{t}=\frac{\sum_{q=0}^{s-1}\left|\Delta w_{i j}^{k}(t-q \Delta t)\right|}{s} \quad \forall t
$$

averaging over $s$ time-increments of $\Delta t$. The length of the time-increments averaged over is related to the time-scale of interest, a parameter similar to the length of period to be considered as stationary in any systems analysis.

\section{STATIONARY PERIOD}

In biology, $s \Delta t$ can be a short time period that corresponds to physiological time-scale (which spans a stimulusresponse episode), a longer time period (which spans multiple trials of stimulus-response cycles) or even the lifetime of an animal.

\section{ITERATIVE SEARCH FOR STABILITY}

In simulations, this parameter $s$ can be derived adaptively using iterative methods, starting with a small number, and then compute the moving-average with an increasing $s$ until the system reaches a stability state as established by the stability criterion.

\section{STABILITY CRITERION}

If this moving-average is approximately a small constant over time, then the system can be considered as approaching a stable state, since the average weight-change is small:

$|\Delta \bar{w}|_{t} \leq \varepsilon$

where $\varepsilon$ is a small constant. The stability criterion, $\varepsilon$, is also an implementation-specific parameter on the limits of fluctuation for the system.

\section{SYSTEM RESTART CRITERION}

When the system reaches a steady-state, whether this stable state is a candidate solution for the system depends on the survivability fitness-test for the system in the evolutionary process. That is because a system can be stuck at a stable state (local minimum) that may not correspond to a real world solution to the problem (global minimum).

If it happens to be an inappropriate (or invalid) solution, the system would fail to interact with the environment appropriately, and it will be eliminated in the survivability test. When this happens, the system dies. A new initial condition will be used to restart the system for another round of evolution.

\section{FIXATION OF CIRCUITRY BY FREEZING THE LEARNING RATE}

Once the system arrives at a stability state, the circuitry can be fixated into hardwire rather than allowing it to continue to change and modified. In other words, the learning coefficient, $l$, described in Eq. 30, representing the weightchange learning rule is no longer a constant, but will approach zero when the system reaches a stable state.

So the learning parameter, $l$, can now be changed with time such that $l(t)$ is dependent on the overall weight-change average:
$l(t)=f^{\prime}\left(|\Delta \bar{w}|_{t}\right)$

and from Eq. 16b:

$l^{\prime}(t)=l(t) / \Delta t$

where $f^{\prime}(\cdot)$ is a function that can be a simple proportional linear function or other nonlinear function, depending on how the system is designed to approach this stability state.

\section{CRITERION FOR FIXATION RULE OF LEARNING}

Eq. 33 shows that, as the moving-average $|\Delta \bar{w}|_{t}$ approaches zero, the learning-rate $l^{\prime}(t)$ will approach zero also. This satisfies the condition for fixation of the circuitry into hardwire without the ability to be modified.

Thus, the final generalized equation for the weightchange is given by:

$\Delta w_{i j}^{k}(t)=l(t) \cdot r \cdot x_{m}^{k}(t) \cdot y_{j}^{k}(t) \cdot x_{i}^{k}(t) \quad \forall i, j, k, m$

or

$\Delta w_{i j}^{k}(t)=f^{\prime}\left(|\Delta \bar{w}|_{t}\right) \cdot r \cdot x_{m}^{k}(t) \cdot y_{j}^{k}(t) \cdot x_{i}^{k}(t) \quad \forall i, j, k, m$

which encapsulates both connection weight-change learning rule and circuitry fixation rule simultaneously.

\section{CONCLUSION}

The above derivations provide the theoretical framework for establishing the principles for emotional context formation in sensation with minimal assumptions based on evolutionary principles without any a priori knowledge of the environment, the system or what emotion is used for. It uses a self-bootstrap feedforward approach to establish the innate responses and reinforcers, and then consolidates the hardwiring of circuitry by the fitness survival-test feedback.

With the innate reflex circuitry established, associated reinforcement learning is used to transfer the relevant sensory signals into derived reinforcers to form the significance of these inputs by adjusting the individual gains (connection weights, reinforcers and learning rate) of the system.

As a result of the self-selected biases produced by the multiple adaptive gains, the system can respond to the environment within context that enhances the response selectively in respond to those stimuli.

Thus contextual significance of the environmental conditions forms the emotional context in which the system (or animal) responds to. The emotional feel of these sensory stimuli emerges as a result.

\section{SUMMARY}

A comprehensive theoretical framework based on an autonomous control system is introduced in this paper to derive the basic set of principles that encapsulate emotions as the emergent properties for increasing the chance of survival in an environment with a minimal set of assumptions.

The theoretical framework does not rely on retrospective (or introspective) account, experience or artificial construct of what emotions are for, or what the roles of emotions are. It 
does not rely on any innate, hardwired or pre-programmed $a$ priori knowledge of what the system is attempting to accomplish.

The only basic assumption of this autonomous system is the existence of the sensory-motor I/O processing elements that forms a neural network. The other assumption is the existence of feedforward and feedback control. No explicit neural network architecture is assumed either; in fact, the neural network architecture can be self-organized by the feedforward and feedback mechanism to eliminating (or consolidating) connections between neurons by decreasing (or increasing) their connection weights, reinforcers and/or learning rate.

The reinforcers needed for the associative learning are also self-selected and self-generated, with the initial innate reinforcer formed by the feedforward evolutionary process, and subsequent reinforcers established by feedback reinforcement correlated with the contextual environmental input. The innate responses are derived from self-bootstrap methods based on evolution principles such that these innate responses can serve as reinforcing signals without any a priori assumptions on what these reinforcers should be.

Thus, this theoretical framework for contextual emotion formation is self-organizing and self-selecting within an autonomous control system without any external "teacher", without any presumed a priori knowledge of the environment, or what the autonomous system is expected to behave or "feel".

The sensory input, the output actions and the internal representation of the external environment are encapsulated by the implicit model, which is created by the neural network processing circuitry. The emotional context of the sensorimotor actions (including reflex actions, conditioned reflexes, and conditioned emotional responses) is the emergent property of this self-organizing neural network.

This represents the first phase of an emotional model called the "EMOTION-I" model, which focuses on the minimal sets of principles for establishing emotional context in sensation. The next phase of the "EMOTION-II" model presented in the next paper [5] will establish the "internal model" needed for the representation of the external world for an autonomous control system to function, and provide the derivation of principles for the emergence of happy/unhappy emotions for self-assessment and consistency-check when comparing the discrepancy between the expectancy in objective and subject realities (not just sensation, as in EMOTION-I).

\section{REFERENCES}

[1] S. C. Gadanho, "Learning Behavior-Selection by Emotions and Cognition in a Multi-Goal Robot Task," J. Mach. Learn. Res., vol. 4, pp. 385-412, Dec 2003.

[2] S. C. Gadanho and J. Hallam, "Emotion-triggered learning in autonomous robot control," Cybernetics and Systems, vol. 32, pp. 531-559, Jul 2001.

[3] A. Takanishi, K. Sato, K. Segawa, H. Takanobu, and H. Miwa, "An Anthropomorphic Head-Eye Robot Expressing Emotions Based on Equations of Emotion," Proc. IEEE Int. Conf. Robot. Automat., vol. 3, pp. 2243-2249, Nov-Dec 2000.
[4] M. A. Arbib, "Evolving emotions in animal and robot," Int. J. Computat. Intel. Applicat., vol. 4, pp. 225-236, Sept, 2004.

[5] D. Tam, "EMOTION-II Model: A Theoretical Framework for Happy Emotion as a Self-Assessment Measure Indicating the Degree-of-Fit (Congruency) between the Expectancy in Subjective and Objective Realities in Autonomous Control Systems," The Open Cybernetics \& Systemics Journal, vol. 1, pp. 47-60, Dec 2007. [Online] Available: http://www.bentham.org/open/tocsj/.

[6] V. Castellucci, H. Pinsker, I. Kupfermann, and E. R. Kandel, "Neuronal mechanisms of habituation and dishabituation of the gillwithdrawal reflex in Aplysia," Science, vol. 167, pp. 1745-8, Mar 1970 .

[7] I. Kupfermann, V. Castellucci, H. Pinsker, and E. Kandel, "Neuronal correlates of habituation and dishabituation of the gillwithdrawal reflex in Aplysia," Science, vol. 167, pp. 1743-5, Mar 1970 .

[8] H. Pinsker, I. Kupfermann, V. Castellucci, and E. Kandel, "Habituation and dishabituation of the gill-withdrawal reflex in Aplysia," Science, vol. 167, pp. 1740-2, Mar 1970.

[9] M M. B. Arnold, "Emotion, motivation, and the limbic system," Ann. N. Y. Acad. Sci., vol. 159, pp. 1041-1058, Jul 1969.

[10] D. A. McCormick, D. G. Lavond, and R. F. Thompson, "Neuronal responses of the rabbit brainstem during performance of the classically conditioned nictitating membrane (NM)/eyelid response," Brain Res., vol. 271, pp. 73-88, Jul 1983.

[11] A. G. Barto and R. S. Sutton, "Landmark learning: an illustration of associative search," Biol. Cybern., vol. 42, pp. 1-8, Nov, 1981.

[12] A. G. Barto, C. W. Anderson, and R. S. Sutton, "Synthesis of nonlinear control surfaces by a layered associative search network," Biol. Cybern., vol. 43, pp. 175-185, Apr, 1982.

[13] R. S. Sutton and A. G. Barto, "Toward a modern theory of adaptive networks: expectation and prediction," Psychol. Rev., vol. 88, pp. 135-70, Mar 1981.

[14] M. L. Commons, S. Grossberg, and J. E. R. Staddon, "Neural network models of conditioning and action", Quantitative analyses of behavior (Unnumbered), Hillsdale, N.J.: L. Erlbaum Associates, 1991.

[15] J. A. Anderson, An introduction to neural networks. Cambridge, Mass.: MIT Press, 1995.

[16] P. R. Montague and T. J. Sejnowski, "The predictive brain: temporal coincidence and temporal order in synaptic learning mechanisms," Learning \& memory (Cold Spring Harbor, N.Y.), vol. 1, May-Jun 1994

[17] D. O. Hebb, The organization of behavior; a neuropsychological theory. New York: Wiley, 1949.

[18] D. E. Rumelhart, J. McClelland, and P. R. Group, Parallel distributed processing explorations in the microstructure of cognition. Volume I, Foundations. Cambridge, Ma.; London: MIT Press, 1986.

[19] D. C. Tam, "Computation of cross-correlation function by a timedelayed neural network", in Intelligent Engineering Systems through Artificial Neural Networks, C. H. Dagli, L. I. Burke, B. R. Fernández, J. Ghosh, Eds., American Society of Mechanical Engineers Press, New York, NY, vol. 3, pp. 51-55, 1993.

[20] D. Tam, "Theoretical analysis of cross-correlation of time-series signals computed by a time-delayed Hebbian associative learning neural network," The Open Cybernetics \& Systemics Journal, vol. 1, pp. 1-4, Jul 2007. [Online] Available: http://www.bentham.org/ open/tocsj/

[21] I. P. Pavlov, "Conditioned reflex," Feldsher Akush, vol. 10, pp. 310, Oct 1951.

[22] I. P. Pavlov, "Conditioned reflex," Feldsher Akush, vol. 11, pp. 612, Nov1951.

[23] J. M. van Rossum, C. L. Broekkamp, and A. J. Pijnenburg, "Behavioral correlates of dopaminergic function in the nucleus accumbens," Adv. Biochem. Psychopharmacol., vol. 16, pp. 201-207, 1977.

[24] D. J. Woodward, J.-Y. Chang, P. Janak, A. Azarov, and K. Anstrom, "Part I. Functional Organization of the Ventral Striatopallidal System - Mesolimbic Neuronal Activity across Behavioral States," Ann. N. Y. Acad. Sci., vol. 877, p. 91, Jun 1999.

[25] B. G. Hoebel, "Brain neurotransmitters in food and drug reward," Am. J. Clin. Nutr., vol. 42, pp. 1133-50, Nov 1985.

[26] R. A. Wise, "The role of reward pathways in the development of drug dependence," Pharmacol. Ther., vol. 35, pp. 227-263, 1987. 
[27] R. A. Wise and M. A. Bozarth, "Brain mechanisms of drug reward and euphoria," Psychiatr. Med., vol. 3, pp. 445-460, 1985.

[28] I. Aharon, N. Etcoff, D. Ariely, C. F. Chabris, E. O'Connor, H. C. Breiter. "Beautiful faces have variable reward value: fMRI and behavioral evidence", Neuron, vol. 32, pp. 537-551, Nov 2001.

[29] R. A. Wise, "Neural mechanisms of the reinforcing action of cocaine," NIDA Res. Monogr., vol. 50, pp. 15-33, 1984.

[30] C. G. Langton, "Artificial life", Addison-Wesley, Redwood City, Calif., 1987.

[31] R. G. Phillips and J. E. LeDoux, "Differential contribution of amygdala and hippocampus to cued and contextual fear conditioning," Behav. Neurosci., vol. 106, pp. 274-85, Apr 1992.
[32] J. LeDoux, "The emotional brain, fear, and the amygdala," Cell. Mol. Neurobiol., vol. 23, pp. 4-5, Oct 2003.

[33] S. C. Gadanho, J. Hallam, "The role of emotions exploring autonomy mechanisms in mobile robots", D.A.I. research paper, no. 851 . Edinburgh: University of Edinburgh, Dept. of Artificial Intelligence, 1997.

[34] S. C. Gadanho, J. Hallam, "Emotion-driven learning for animat control", D.A.I. research paper, no. 881. Edinburgh: University of Edinburgh, Dept. of Artificial Intelligence, 1998. 